\title{
TD-LTE Private Network Performance Analysis in Smart Grid
}

\author{
Ying Sun ${ }^{1,2, a^{*}}$, Xuanni Lin ${ }^{1, b}$, Yuqing Zhong ${ }^{1, c}$ \\ ${ }^{1}$ Guangzhou Power Supply Co. Ltd., CSG, Guangzhou 510620, China \\ ${ }^{2}$ Shenzhen Graduate School of Harbin Institute of Technology, Shenzhen 518055, China

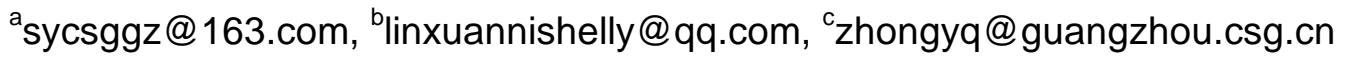

Keywords: private network; TD-LTE; spectral efficiency; energy efficiency

Abstract. Smart Grid applications require an efficient, reliable and robust private communication network to achieve the ubiquitous and mutual transmission of power grid data such as distribution automation, smart metering and video surveillance. This paper investigates the wide-spread deployment of TD-LTE private network in Smart Grid, presenting its system architecture and network implementation. Two important parameters, namely, spectral efficiency and energy efficiency, are then introduced to evaluate the system performance. Furthermore, detailed analysis and suggestions are provided for the deployment and implementation of TD-LTE private network in the presence of inter-cell interference, thus achieving the energy and cost efficient private wireless communication network for Smart Grid.

\section{Introduction}

Smart Grid has been witnessing the rapid deployment of TD-LTE private network to accommodate the specific requirements of power industry such as stability, reliability, safe differentiated authority control and clustering communication[1]. In return, the various wireless network applications of distribution automation, advanced metering, electric vehicle and video surveillance have been speeding up the development of TD-LTE private network towards a robust, large-capacity, low-energy-consumption communication network[2].

Currently, TD-LTE private networks in China are mainly implemented on two frequency bands: $1.8 \mathrm{GHz}$ and $230 \mathrm{MHz}[3]$. The former band provides broad bandwidth and thus accommodates the applications of distribution automation, video surveillance, etc, which is more suited for the areas with dense sites and high traffic demands. The latter band, on the other hand, provides large coverage and is often used in the coverage scenario of distributed sites and sparse traffic demands such as smart metering automation. However, TD-LTE private network, either in $1.8 \mathrm{GHz}$ or $230 \mathrm{MHz}$, is facing the problem of scarce spectrum resource and high capital and operational expenditure (CAPEX and OPEX), especially the high electricity bills[4], thus limiting its system performance and long-term development.

Considering the deficiencies above, this paper analyzes the system performance of TD-LTE private network in Smart Grid, thus providing suggestions and references for its deployment in a variety of power grid communication applications. TD-LTE private network architecture is presented, including the traffic demand, frequency band choice and deployment mode. Then, spectral efficiency (SE) and energy efficiency (EE) are introduced to evaluate the utilization of frequency and energy resources. Moreover, the system performance in single-cell and double-cell coverage scenarios with the presence of inter-cell interference is investigated.

\section{Network Architecture}

TD-LTE private network consists of three layers, namely, terminal layer, radio access layer and core network layer[5], as illustrated in Fig.1. Mobile terminal layer consists of access terminals including fixed and handheld terminals, laptops, USB data cards, etc. Radio access layer consists of baseband units (BBU), remote radio units (RRU) and gateways, which provide radio signals, resource 
management, service control functions for terminals. Core network layer consists of service and network management systems, which maintain the whole network in a centralized way.

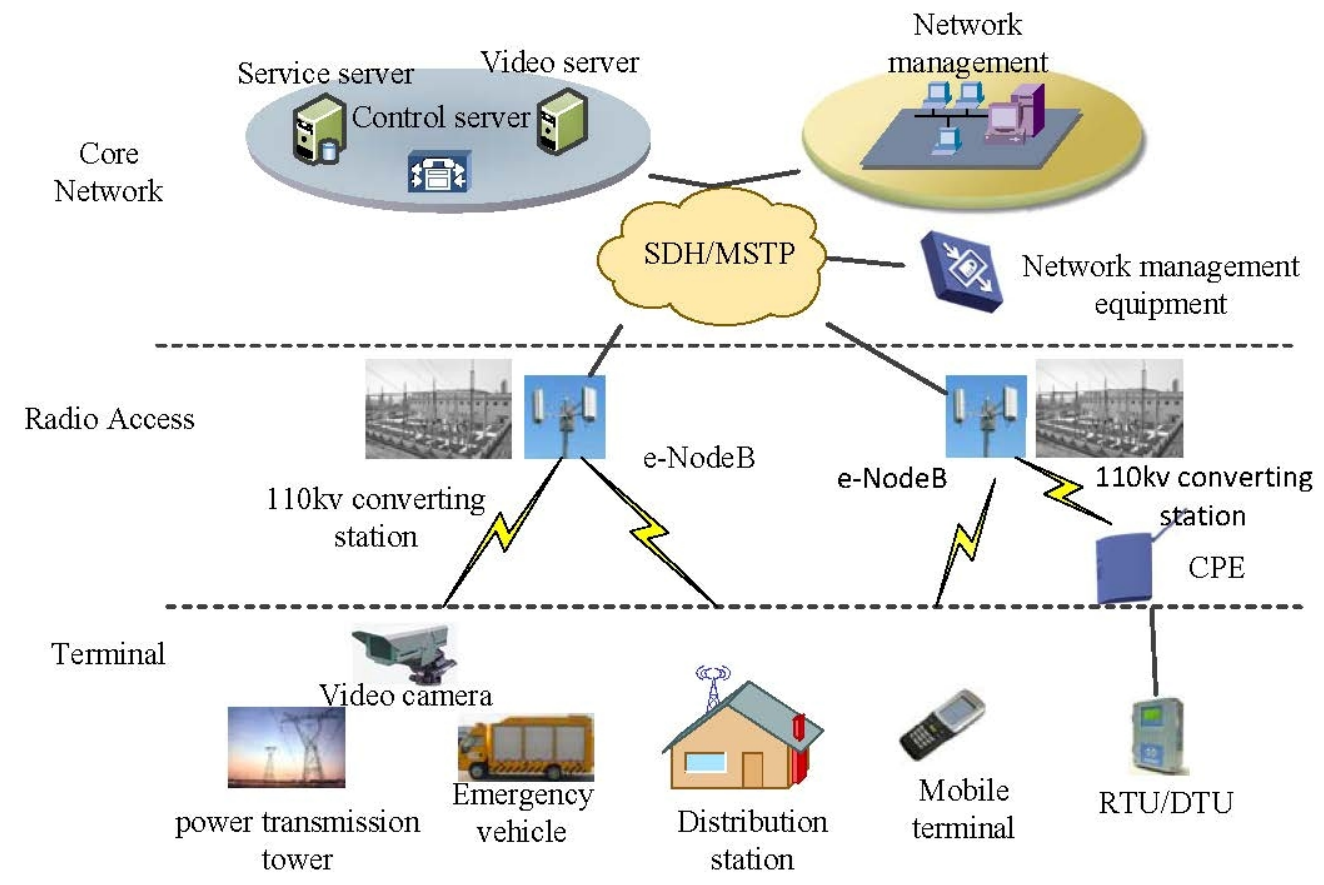

Figure 1. TD-LTE private network architecture.

Service Requirements. TD-LTE private network could accommodate various Smart Grid data applications, such as distribution automation, user service, electricity information metering, payload management, emergency situations, all of which have different requirements on the network performance.

Distribution Automation. Distribution automation is one of the essential services in smart power distribution and provides automatic monitoring and control for distribution switch and ring main unit, which could achieve reconstruction of distribution network and enhance its reliability. The distribution automation services require the existence of $2.4 \mathrm{kbps}$ wireless full duplex communication channel between every distribution terminal and stations.

User Service. Smart Gird reaches the terminal users through communication network, thus forming the home area network (HAN) and providing users' electricity information, transaction information and smart electricity management function, all of which require a channel of at least $0.3 \mathrm{kbps}$ for each user. Advanced Metering. Advanced metering collects the electricity information of public voltage transformer, low voltage industrial and community users to provide power line loss assessment and pre-paid service management, which needs the existence of $19.2 \mathrm{kbps}$ wireless full duplex channel between the public voltage transformer and metering station.

Payload management. Payload management monitors the electricity usage of private voltage transformer (around $100 \mathrm{kVa}$ ), thus facilitating the power usage management and information metering, which requires the existence of $1.2 \mathrm{kbps}$ wireless full duplex channel between each terminal and metering station.

Emergency situations. In case of emergency, urgent repair, recondition, etc., power grid data need to be delivered in a visualized way with the integration of Global Positioning System (GPS), thus requiring $2 \mathrm{Mbps}$ bandwidth for each terminal to achieve the capital and positioning management of mobile terminals.

As can be seen from the above applications, the services of TD-LTE private network demonstrate apparent power industry characteristics. For instance, public wireless network often focuses on downlink services while the TD-LTE private network has higher requirements on uplink transmission data rate due to the metering services. 
Assuming a single metering station exchanges data with 1200 smart meters, each of which transmits $5 \mathrm{kbits}$ packet in $60 \mathrm{~s}$, the data transmission rate between each smart meter and the station is calculated as follows:

$$
R_{\mathrm{SM}}=\sum_{i} R_{\mathrm{SM}}^{i}=1 \mathrm{Mbps}
$$

Moreover, assuming the metering station connects to 10 video surveillance cameras, each of which requires $512 \mathrm{kbps}$ rate, the data transmission rate between the metering station and the cameras is calculated as follows:

$$
R_{\mathrm{SU}}=\sum_{i} R_{\mathrm{SU}}^{i}=5 \mathrm{Mbps}
$$

There are two frequency bands available in China for TD-LTE private network, namely, $230 \mathrm{MHz}$ and $1.8 \mathrm{GHz}$. The former has 40 distributed frequency points allocated to power grid, all of which occupy a total of $1 \mathrm{MHz}$ bandwidth. Meanwhile, additional 3-4MHz bandwidth could be obtained by sharing with other industries. The latter is the globally popular frequency choice of Smart Grid communication with some bandwidth $(5 \mathrm{MHz})$ is also allocated to power grid in China.

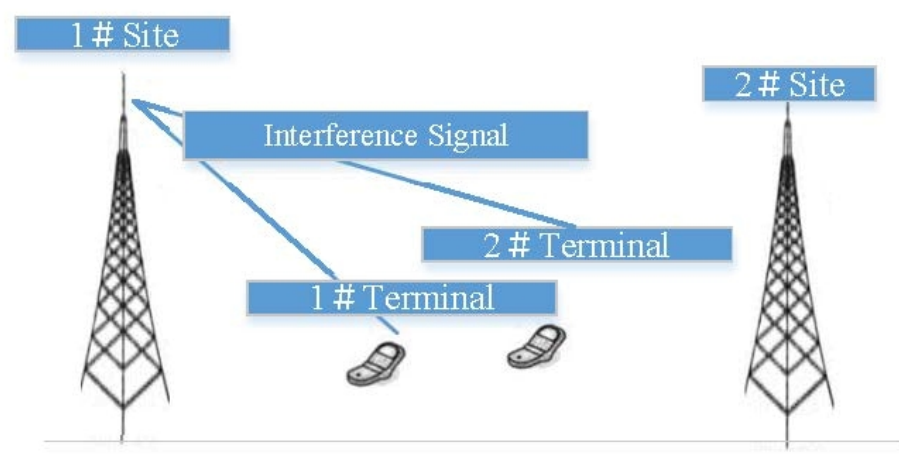

Figure 2. Illustration of downlink interference in TD-LTE private network.

\section{Deployment Mode}

TD-LTE private network could be deployed with or without frequency reuse.

Frequency Sharing. In this mode, all cells could use the same frequency band to provide services for terminals in the coverage areas, thus utilizing frequency resource efficiently. However, inter-cell interference exists between neighboring cells, in which e-NodeBs interfere cell edge terminals in downlink while cell edge terminals interfere neighboring e-NodeBs in uplink, as illustrated in Fig.2 and Fig.3.

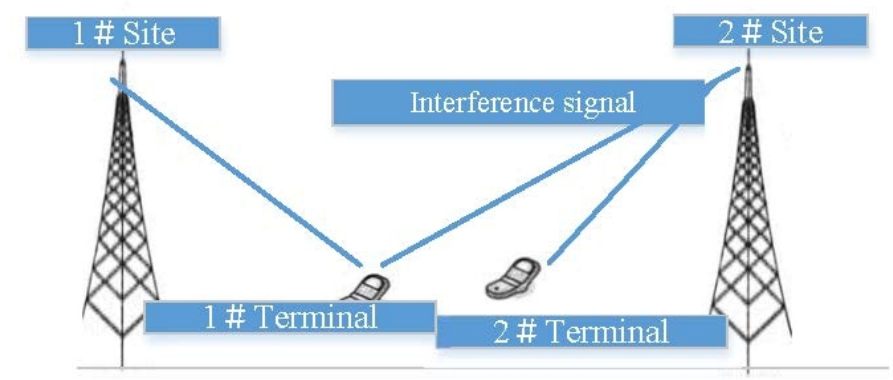

Figure 3. Illustration of uplink interference in TD-LTE private network.

Frequency Reuse. In this mode, neighboring cells use different frequency points to mitigate interference and improve transmission rate of cell-edge users. However, the improvement is achieved at the price of inadequate frequency utilization. To trade-off the system performance and frequency utilization, two frequency reuse modes are often used, namely, four-color and six-color, as shown in Fig.4 and Fig.5. 


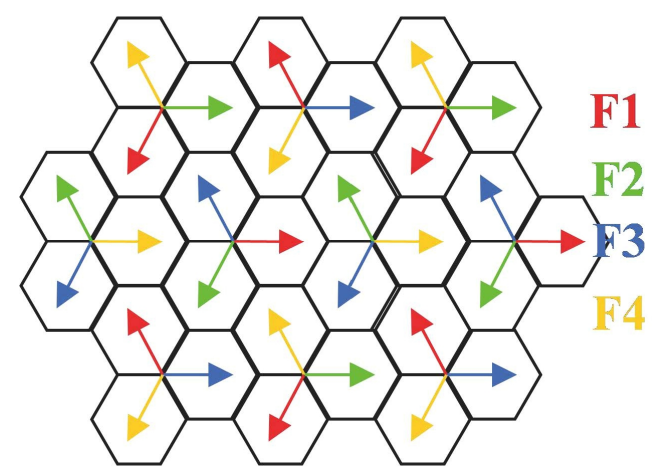

Figure 4. Illustration of four color frequency reuse.

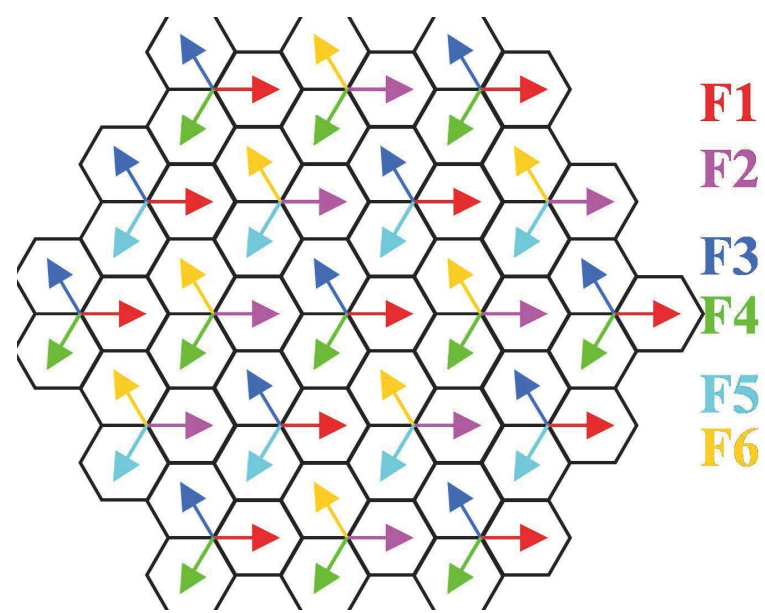

Figure 5. Illustration of six color frequency reuse.

Considering the broadband requirements of Smart Grid appliations such as smart metering, video surveillance in a very limited frequency band, TD-LTE private network needs to improve bandwidth utilization and reduce power consumption to achieve higher system capacity and lower the OPEX. Therefore, frequency sharing mode, i.e., frequency reuse factor is 1 , is prefered.

\section{Summary}

This paper investigated the bandwidth utilization and power consumption problem in TD-LTE private network to meet the increasing demands of Smart Grid communication applications. Detailed analysis on the service requirements, frequency choice, deployment mode, etc was given to obtain the network architecture and thus provide suggestions for future TD-LTE private network deployment.

\section{References}

[1] X. Li, Q. He, and J. Sun, Research and application on communication technology in smart distribution and utilization grid, Electric Power, 12(1):1-6, 2011.

[2] Y. Sun, Y. Huang, L. Chen, A centralized LTE private wireless network architecture for smart grid communication network, Appl. Mech. Mater., 687(1):2363-2366, 2014.

[3] Y. Sun, X. Lin, L. Chen, Utilizing indicator computing assistant to facilitate distribution automation in LTE wireless private network, Appl. Mech. Mater., 691(1):3239-3242, 2014.

[4] Y. Chen, S. Zhang, S. XU, et al., Fundamental trade-offs on green wireless networks, IEEE Communications Magazine, 49(6):30-37, 2011.

[5] D. Lopez-Perez, I. Guvenc, G. de la Roche, M. Kountouris, T.Q.S. Quek, J. Zhang, Enhanced intercell interference coordination challenges in heterogeneous networks, IEEE Wireless Communications, 18(3):22-30, 2011. 